\title{
Rare presentation of Basedow' disease with myalgia: A case report
}

\author{
Masaru Kurihara $^{1}$, Shunichi Kinjo ${ }^{1}$, and Yasuharu Tokuda ${ }^{2}$ \\ ${ }^{1}$ Urasoe General Hospital \\ ${ }^{2}$ Muribushi Okinawa Center for Teaching Hospitals
}

April 19, 2021

\begin{abstract}
A 42-year-old woman presented with myalgia, which ameliorated a week after treatment. She was diagnosed with Basedow's disease. The presence of concomitant autoimmune diseases are important considerations for patients with Basedow's disease presenting with myalgia. Thyrotoxicosis should be included as a rare differential diagnosis for myalgia.
\end{abstract}

\section{Introduction}

Basedow's disease is the most common cause of thyrotoxicosis. ${ }^{1}$ Thyrotropin receptor antibody (TRAb) is produced on the surface of the thyroid gland as an autoantibody against the binding receptor of thyroid stimulating hormone (TSH). TRAb overstimulates the TSH receptor instead of TSH, causing excessive production of thyroid hormones that subsequently causes Basedow's disease. ${ }^{1}$ Abnormally high levels of thyroid hormones that increase the metabolism of the entire body can have significant health consequences. Known autoimmune mechanisms include orbital disease, goitre, and thyroid dermatosis. ${ }^{2}$ Symptoms of Basedow's disease can be caused by either hyperthyroidism or autoimmune mechanisms. Hypothyroidism commonly causes myalgia in thyroid gland dysfunction. ${ }^{3}$ Herein, we report a case of Basedow's disease that presented with a rare complaint of myalgia.

\section{Case history}

A 42-year-old Japanese woman presented with a 4-week history of myalgia. Two weeks before her visit to our hospital, her pain began to spread from the neck to the chest, resulting in extreme myalgia four days prior to her visit. The pain was sudden, non-persistent, and generalised with no clear localisation. There was no change in her symptoms owing to diet. The patient reported excessive sweating and weight loss $(5$ $\mathrm{kg} / 6$ months); however, tremors, diarrhoea, and diplopia were absent. She had no remarkable family history, though her medical history included pyelonephritis. Furthermore, she was not taking any oral medications or supplements.

On examination, the patient appeared ill and was afebrile $\left(37.0{ }^{\circ} \mathrm{C}\right)$. Her blood pressure was $110 / 64 \mathrm{mmHg}$, pulse $90 / \mathrm{min}$, respiratory rate $16 / \mathrm{min}$ with an $\mathrm{O}_{2}$ saturation of $98 \%$ on room air. Physical examination revealed no ocular protrusion or eye movement disorder. Her thyroid gland was diffusely enlarged, but there was no associated tenderness or nodule present. Her cardiac examination results were normal, and lungs were clear on auscultation. There were no abnormal abdominal findings. Findings of neurological examinations, including tests for muscle weakness, muscle grasping pain, muscle atrophy, and deep tendon reflexes, were completely unremarkable.

On admission, initial investigations revealed mild anaemia. Inflammatory markers and kidney function test results were normal, but liver function test values were mildly elevated. Creatinine kinase, serum calcium, and serum phosphate levels were normal. Further examination revealed free triiodothyronine $(\mathrm{FT} 3)>30.00$ $\mathrm{ng} / \mathrm{mL}$, free thyroxine (FT4) $3.45 \mathrm{ng} / \mathrm{mL}, \mathrm{TSH}<0.01 \mu \mathrm{IU} / \mathrm{mL}$, and findings of thyrotoxicosis. Additionally, 
she tested positive for TRAb (6.4 IU/L). The results of tests for autoantibodies, including antinuclear antibodies, were negative (Table 1 ).

Cervical ultrasonography showed diffuse enlargement of the thyroid gland and increased blood flow with a colour Doppler scan of $57.6 \mathrm{~cm} / \mathrm{s}$ and $48.1 \mathrm{~cm} / \mathrm{s}$ on the right and left, respectively. Consequently, she was diagnosed with Basedow's disease. Since there were no specific symptoms and autoantibodies were absent, we concluded that there was no complication of myositis. Treatment with mercazole $15 \mathrm{mg}$ and potassium iodide $50 \mathrm{mg}$ was initiated, leading to an improvement in FT3 $(>4.49 \mathrm{ng} / \mathrm{mL})$ and FT4 $(1.31 \mathrm{ng} / \mathrm{mL})$ levels after one month of treatment. Myalgia disappeared one week after the administration of the anti-thyroid medication. Thereafter, the condition of the patient improved without any relapse of pain.

\section{Discussion}

Our patient had Basedow's disease and presented with the rare chief complaint of myalgia. Musculoskeletal symptoms and signs are common in patients with thyroid dysfunction, as the skeletal muscle is a major target of thyroid hormones. ${ }^{4}$ Although hypothyroidism is a thyroid function abnormality associated with myalgia, hyperthyroidism is mainly associated with myopathy, such as muscle weakness and wasting. Symptoms of myopathy primarily involve the proximal muscles and rarely the pectoralis major muscle. ${ }^{5}$ Hyperthyroid myopathy usually resolves after recovery from hyperthyroidism. In the present case, the findings were atypical because there was no muscle weakness, no muscular symptoms, and myalgia was not localised to the muscles. Since myalgia in cases such as the presented one is rare, careful consideration of the complications of concomitant disease is needed, followed by a detailed medical interview, review of the system, and systemic physical examination.

In recent years, cases of myalgia and elevated creatine kinase levels during the treatment of hyperthyroidism have been presented, and the side effects of anti-thyroid drugs and relative hypothyroidism have been proposed as explanations for these muscle symptoms. ${ }^{5}$ However, the presented case is not consistent with relative hypothyroidism because myalgia was present before treatment. Because T4 abnormalities cause abnormal glycogen degradation, abnormal mitochondrial oxidative metabolism, and abnormal triglyceride turnover, which impair muscle function, ${ }^{6}$ it is usually seen in hypothyroidism, but may be affected in hyperthyroidism as well. Muscle symptoms caused by Basedow's disease require attention as they could be complications of other autoimmune diseases. Basedow's disease is rarely associated with other autoimmune diseases, especially polymyositis and myasthenia gravis. ${ }^{7,8}$ Further, the absence of other features of muscle fatigue and myasthenia gravis in the present case ruled out other autoimmune diseases. Similarly, periodic limb paralysis is a rare and life-threatening complication of Basedow's disease. ${ }^{9}$ It is characterised by recurrent episodes of transient, flaccid muscle paralysis and affects the proximal muscles more severely than distal muscles. The clinical course of the presented case and normal serum potassium levels were inconsistent with periodic quadriplegia. Furthermore, vitamin D deficiency can be an aetiological factor for non-specific musculoskeletal pain. ${ }^{10}$ However, this possibility was ruled out because there was no evidence of vitamin D deficiency in the present case. As in previous reports, ${ }^{11}$ the improvement in myalgia as well as the simultaneous recovery of hyperthyroidism strongly suggests that hyperthyroidism was the cause of the myalgia. To the best of our knowledge, this is a very rare symptom of Basedow's disease and has only been reported in two previous cases. ${ }^{9}$

In conclusion, we report on a patient with Basedow's disease who presented with the chief complaint of myalgia, which is a rare symptom. In patients with Basedow's disease who complain of muscular symptoms, it is important to consider the disease mechanism as well as the presence of other concomitant diseases; thyrotoxicosis could be a differential diagnosis in such patients who present with myalgia.

Informed consent: We obtained informed consent from the patient for publication of this case report.

Data accessibility: The datasets used and/or analyzed during the current study are available from the corresponding author on reasonable request.

Acknowledgments: None. This study did not receive any funding. 
Funding: None. This study did not receive any funding.

An ethics statement: We have obtained informed consent from the patient for publication of this case report.

Conflict of Interest : None. We had no Conflict of Interest.

Author Contributions:

All authors critically revised the report, commented on drafts of the manuscript, and approved the final report.

\section{References}

1. Bahn, R. 2012. Autoimmunity and Graves' disease. Clin Pharmacol Ther 91:577 -579 .

2. Bartalena, L., and V. Fatourechi. 2014. Extrathyroidal manifestations of Graves' disease: a 2014 update. J Endocrinol Invest 37:691-700.

3. Lu, R., H. Wang, T. Hong, and H. Gao. 2020. Myopathy after rapid correction of hyperthyroidism: a case report and review of literature. Medicine 99:e18878.

4. Salvatore, D., W.S. Simonides, M. Dentice, A.M. Zavacki, and P.R. Larsen. 2013. Thyroid hormones and skeletal muscle - new insights and potential implications. Nat Rev Endocrinol 10:206-214.

5. Mathew B., A.J. Devasia, V. Ayyar, V. Thyagaraj, and G.A. Francis. 2011. Thyrotoxicosis presenting as acute bulbar palsy. J Assoc Phys India 59:386-387.

6. Argov Z., and D.L. Arnold. 2002. MR spectroscopy and MR imaging in metabolic myopathies. Neurologic Clinics 18:35-52.

7. Wang H., H. Li, C. Kai, and J. Deng. 2010. Polymyositis associated with hypothyroidism or hyperthyroidism: two cases and review of the literature. Clin Rheumatol 30:449-458.

8. Masood I., M. Yasir, A. Aiman, and R.P. Kudyar. 2009. Autoimmune thyroid disease with myasthenia gravis in a 28-year-old male: a case report. Cases J 2:8766.

9. Hong S.B., Y.J. Park, Y.J. Oh, Y.W. Kim, S.K. Kim, M.R. Kim, et al. 2001. Graves' disease detected by myalgia. (In Korean.) Korean J Med 61:173-177.

10. Glueck C.J., and B. Conrad. 2013. Severe vitamin D deficiency, myopathy, and rhabdomyolysis. North Am J Med Sci 5:494-495.

11. Papanikolaou N., and P. Perros. 2013. An unusual presenting symptom of Graves' disease: myalgia. Eur Thyroid J 1:274-276.

Table. 1 Laboratory Data

\begin{tabular}{lll}
\hline Variable & Reference Range & On Admission \\
\hline Haemoglobin $(\mathrm{g} / \mathrm{dL})$ & $11.6-14.8$ & 10.8 \\
Haematocrit $(\%)$ & $35.1-44.4$ & 33.5 \\
White blood cell count $($ per $\mu \mathrm{L})$ & $3300-8600$ & 3500 \\
Differential count (per $\mu \mathrm{L})$ & & \\
Neutrophils (\%) & $0.0-2.5$ & 39.0 \\
Lymphocytes (\%) & $16.5-49.5$ & 43.8 \\
Monocytes (\%) & $2.0-10.0$ & 14.1 \\
Eosinophil (\%) & $0.0-8.5$ & 2.8 \\
Platelet count (per $\mu \mathrm{L})$ & $158000-348000$ & 221000 \\
Sodium $(\mathrm{mmol} / \mathrm{L})$ & $135-145$ & 141 \\
Potassium $(\mathrm{mmol} / \mathrm{L})$ & $3.6-4.8$ & 4.2
\end{tabular}




\begin{tabular}{lll}
\hline Variable & Reference Range & On Admission \\
\hline Chloride $(\mathrm{mmol} / \mathrm{L})$ & $101-108$ & 109 \\
Calcium $(\mathrm{mg} / \mathrm{dL})$ & $8.8-10.1$ & 9.1 \\
Inorganic phosphate $(\mathrm{mg} / \mathrm{dL})$ & $2.4-4.3$ & 2.7 \\
Urea nitrogen $(\mathrm{mg} / \mathrm{dL})$ & $8.0-20.0$ & 14.7 \\
Creatinine $(\mathrm{mg} / \mathrm{dL})$ & $0.46-0.79$ & 0.49 \\
Alanine aminotransferase (U/L) & $13-30$ & 50 \\
Aspartate aminotransferase (U/L) & $7-23$ & 93 \\
Alkaline phosphatase (U/L) & $106-322$ & 217 \\
Creatine kinase (U/L) & $41-153$ & 138 \\
Lactate dehydrogenase (U/L) & $124-222$ & 194 \\
C-reactive protein $(\mathrm{mg} / \mathrm{dL})$ & $0.0-0.1$ & 0.0 \\
FT3 (ng/mL) & $1.71-3.71$ & $>30.0$ \\
FT4 (ng/mL) & $0.70-1.48$ & 3.45 \\
TSH ( $\mu$ IU/mL) & $0.35-4.94$ & $<0.01$ \\
TRAb (IU/L) & $<2.0$ & 6.4 \\
Anti-thyroglobulin antibody & $<28.0$ & 383.0 \\
Anti-thyroid peroxidase & $0.0-15.9$ & 14.3 \\
antibodies & $<180$ & 280 \\
TSAb (\%) & $<45$ & 90.0 \\
Thyroglobulin (ng/mL) & $<40$ & negative \\
Antinuclear antibody & $<25$ & negative \\
Anti-aminoacyl tRNA & & \\
synthetase antibody & $0.0-15.0$ & 0.0 \\
Rheumatoid factor (U/mL) & &
\end{tabular}

Abbreviations: FT3, free triiodothyronine; FT4, free thyroxine; RNA, ribonucleic acid; TRAb, thyrotropin receptor antibody; TSH, thyroid stimulating hormone (thyrotropin); TSAb, thyroid stimulating antibody. 